\title{
Tumor PD-L1 expression is associated with outcomes in stage III non-small cell lung cancer (NSCLC) patients treated with consolidation durvalumab
}

\author{
Khalid Jazieh $^{1}$, Mohamed Gad ${ }^{1}$, Anas Saad ${ }^{2}$, Wei Wei ${ }^{3}$, Nathan A. Pennell ${ }^{4}$ \\ ${ }^{1}$ Department of Internal Medicine, Cleveland Clinic Foundation, Cleveland, OH, USA; ${ }^{2}$ Heart and Vascular Institute, Cleveland Clinic Foundation, \\ Cleveland, OH, USA; ${ }^{3}$ Department of Quantitative Health Sciences, Lerner Research Institute, Cleveland Clinic Foundation, Cleveland, OH, USA; \\ ${ }^{4}$ Department of Hematology and Medical Oncology, Taussig Cancer Institute, Cleveland Clinic Foundation, Cleveland, OH, USA \\ Contributions: (I) Conception and design: K Jazieh, NA Pennell; (II) Administrative support: NA Pennell; (III) Provision of study materials or patients: \\ NA Pennell; (IV) Collection and assembly of data: K Jazieh, A Saad, M Gad; (V) Data analysis and interpretation: All authors; (VI) Manuscript \\ writing: All authors; (VII) Final approval of manuscript: All authors. \\ Correspondence to: Khalid Jazieh. Cleveland Clinic Foundation, 9500 Euclid Ave. NA-10, Cleveland, OH 44195, USA. Email: kjazieh@gmail.com.
}

Background: Durvalumab is an anti-PD-L1 immune checkpoint inhibitor approved for consolidation therapy for patients with stage III non-small cell lung cancer (NSCLC) after chemoradiation. The purpose of our study was to evaluate the association between the degree of tumor PD-L1 expression and outcomes of stage III NSCLC patients treated with durvalumab.

Methods: We conducted a retrospective analysis of all the patients who received durvalumab between July 2017 and July 2019 at our facilities and were diagnosed with or progressed to stage III NSCLC before durvalumab consolidation. Patients were divided into groups based on the degree of PD-L1 expression: $<1 \%, 1-49 \%$, and 50-100\%. Overall survival and progression-free survival were estimated by the KaplanMeier method and the Multivariate Cox proportional hazard model was used to assess the effect of PD-L1 expression level on OS and PFS, adjusting for age and gender.

Results: We identified 121 patients with stage III NSCLC that underwent durvalumab consolidation. Of them, 29.8\% had PD-L1 expression of 50-100\%, 24.8\% had PD-L1 expression of 1-49\%, and 27.3\% had PD-L1 expression of $<1 \%$, while $18.2 \%$ were not tested for PD-L1 expression. The rate of cancer progression in the group with $50-100 \%$ PD-L1 expression was $16.7 \%$ compared to $60 \%$ in the $1-49 \%$ expression group and $54.6 \%$ in the $<1 \%$ expression group, and the 1-year survival rates were higher in the $50-100 \%$ group (97\%) compared to the $1-49 \%$ group and the $<1 \%$ group $(73 \%$ and $78 \%$, respectively; $\mathrm{P}=0.028)$. Survival analysis via Kaplan-Meier revealed a significant difference in both $\mathrm{PFS}(\mathrm{P}<0.0001)$ and $\mathrm{OS}$ $(\mathrm{P}<0.028)$ based on the extent of PD-L1 expression. Multivariate analysis revealed that a PD-L1 expression $>50 \%$ was the only factor that was significantly associated with improved PFS (HR $=0.205, \mathrm{P}=0.0004)$ and OS (HR $=0.339, \mathrm{P}=0.04)$.

Conclusions: Our study demonstrated that patients whose tumors had $>50 \%$ PD-L1 expression had significantly longer progression-free survival and overall survival than those with lower PD-L1 expression. This suggests that the degree of tumor PD-L1 expression may play a role in predicting benefit from durvalumab for these patients.

Keywords: Lung cancer; durvalumab; programmed-death ligand 1 (PD-L1)

Submitted Mar 29, 2021. Accepted for publication Jun 04, 2021.

doi: $10.21037 /$ tlcr-21-249

View this article at: https://dx.doi.org/10.21037/tlcr-21-249 


\section{Introduction}

Lung cancer is estimated to be the number one cause of cancer-related deaths in the US in 2021, and non-small cell lung cancer (NSCLC) accounts for about $85 \%$ of lung cancer cases (1). Given the findings of the PACIFIC trial in which durvalumab improved both the progression-free survival (PFS) and overall survival (OS) of patients with stage III NSCLC who had completed chemotherapy and radiotherapy, durvalumab consolidation has now become a mainstay of treatment for patients with stage III NSCLC (2-4). Durvalumab is a monoclonal antibody that targets the programmed-death ligand 1 (PD-L1) on tumor cells, allowing them to be recognized and killed by the immune system $(5,6)$. Testing for PD-L1 expression is typically done via immunohistochemistry (IHC) assays; some of the more common monoclonal antibody clones in testing kits include the 22C3, SP263, and the 28-8 clones (7).

There is little research to indicate the role of PD-L1 expression in predicting outcomes of patients being treated with durvalumab. Several studies indicate that higher PD-L1 expression confers better treatment outcomes in NSCLC patients treated with pembrolizumab $(8,9)$ atezolizumab $(10,11)$ and nivolumab $(12)$, yet other studies have suggested otherwise (13). As for durvalumab, there is data to suggest that PD-L1 expression is associated with higher response rates $(14,15)$. However, the issue with most of these studies is that they are phase I/II with small sample sizes. Additionally, there are different cutoffs used to determine "high" PD-L1 expression. In the PACIFIC trial, patients were divided into groups of those with $<25 \%$ of tumor cells expressing PD-L1 and those with $\geq 25 \%$ PDL1 expression. Improvement in PFS and OS were seen with durvalumab regardless of the extent of PD-L1 expression, although it seemed that the improvement in OS was least noted in patients with $<1 \%$ PD-L1 expression (2-4). The purpose of our study was to evaluate the association between the degree of PD-L1 expression and outcomes of stage III NSCLC patients treated with durvalumab. We present the following article in accordance with the STROBE reporting checklist (16) (available at https://dx.doi.org/10.21037/tlcr21-249).

\section{Methods}

This was a retrospective study of patients with stage III NSCLC treated with durvalumab within the Cleveland Clinic Foundation enterprise. We used our institutional pharmacy records to identify all the patients who received durvalumab between July 2017 and July 2019 at our facilities and selected those who were diagnosed with or progressed to Stage III NSCLC before durvalumab consolidation. Patients who had small cell lung cancer, were treated at stage IV of their disease, or who only received a portion of their care here for a second opinion and then were lost to follow up as they continued their care at other institutions were excluded from our analyses. We decided to select all of the stage III NSCLC patients treated with durvalumab to minimize selection bias and have the most data possible. Information was collected from the patients' electronic medical charts and stored and managed using a secure database between July 2019 and September 2020.

The study was conducted in accordance with the Declaration of Helsinki (as revised in 2013). The study was approved by the institutional review board of the Cleveland Clinic Foundation (NO.: 14-562) and individual consent for this retrospective analysis was waived. Information that was collected about each patient included demographics (age, race, sex), smoking history, clinical and pathological information regarding their cancer (histological subtype, initial stage at diagnosis, T/N/M status, tumor marker expression, PD-L1 expression), treatment course (number of durvalumab doses, side effects), and clinical outcomes.

\section{Statistical analysis}

PFS was defined as the time from initiation of durvalumab until cancer progression (per RECIST criteria) or recurrence was identified (or until the last day durvalumab was known to be effective). OS was defined as the time from initiation of durvalumab until the last day the patient was known to be alive.

Patients were divided into groups based on the degree of PD-L1 expression: < $\%$ (PD-L1 absent), 1-49\% (low expression), and $50-100 \%$ (high expression). The Cleveland Clinic Laboratory performs PD-L1 testing on tissue samples via immunohistochemistry testing for the Dako 22C3 PD-L1 clone to assess the proportion of tumor cells that express PD-L1. Patient characteristics were summarized in median and range for continuous variables, and in frequencies and percentages for categorical variables. Fisher's exact test was used to associate patient characteristics with PD-L1 expression status. Wilcoxon rank-sum test was used to compare age, pack-year, and the number of doses between PD-L1 groups. Overall survival and progression-free survival were estimated by the Kaplan- 
Meier method and were compared using log-rank testing between patient groups. Multivariate Cox proportional hazard model was used to assess the effect of PD-L1 expression level on OS and PFS, adjusting for age and gender. All tests were two-sided and $\mathrm{P}$ values of 0.05 or less were considered statistically significant. Statistical analysis was carried out using SAS Studio 3.7 (SAS Institute, Cary, $\mathrm{NC}$ ) and R 3.6 (R Foundation, Vienna, Austria).

\section{Results}

We identified 138 patients treated with durvalumab at our facilities, of which 121 had stage III NSCLC and fit our aforementioned research criteria. 50.4\% were female, and the mean age was 68.3 years. Of them, 29.8\% had PD-L1 expression of $50-100 \%, 24.8 \%$ had PD-L1 expression of $1-49 \%$, and $27.3 \%$ had PD-L1 expression of $<1 \%$, while $18.2 \%$ were not tested for PD-L1 expression (Table 1). There was no significant difference in age, sex, smoking pack-years, the histologic subtype of NSCLC, and the number of durvalumab doses administered between patients who expressed PD-L1 and those who did not.

Cancer progression occurred in $16.7 \%$ of the patients in the group with $50-100 \%$ PD-L1 expression compared to $60 \%$ in the $1-49 \%$ expression group and $54.6 \%$ in the $<1 \%$ expression group, and the 1-year survival rates were higher

Table 1 Summary of patient characteristics by PD-L1 status

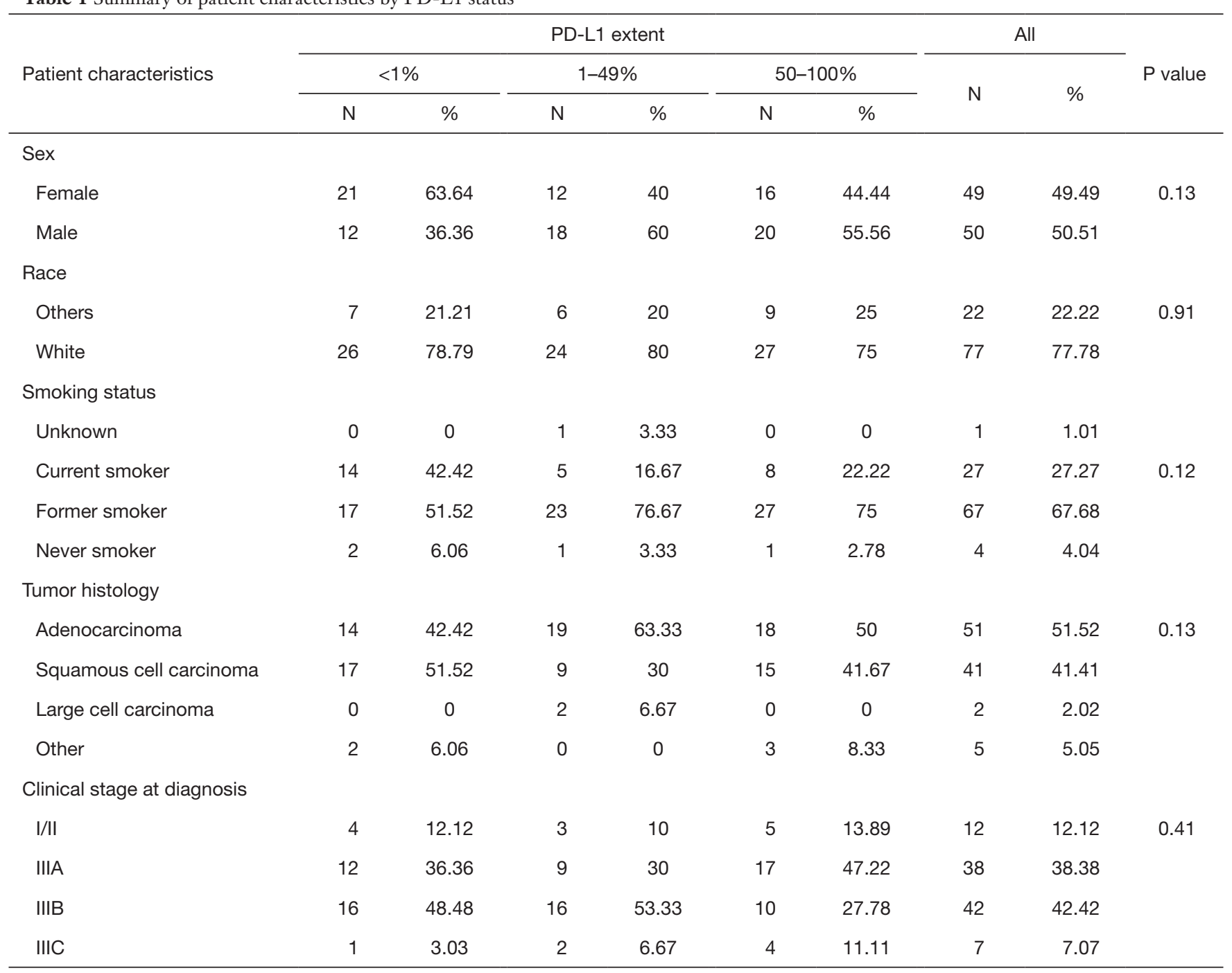

Table 1 (continued) 
Table 1 (continued)

\begin{tabular}{|c|c|c|c|c|c|c|c|c|c|}
\hline \multirow{2}{*}{ Patient characteristics } & \multicolumn{6}{|c|}{ PD-L1 extent } & \multicolumn{2}{|c|}{ All } & \multirow{2}{*}{$P$ value } \\
\hline & $\mathrm{N}$ & $\%$ & $\mathrm{~N}$ & $\%$ & $\mathrm{~N}$ & $\%$ & $\mathrm{~N}$ & $\%$ & \\
\hline \multicolumn{10}{|c|}{ TNM, T status at diagnosis } \\
\hline Unknown & 0 & 0 & 1 & 3.33 & 0 & 0 & 1 & 1.01 & \\
\hline $\mathrm{T} 2$ & 11 & 33.33 & 5 & 16.67 & 9 & 25 & 25 & 25.25 & \\
\hline T3 & 9 & 27.27 & 10 & 33.33 & 9 & 25 & 28 & 28.28 & \\
\hline $\mathrm{T} 4$ & 7 & 21.21 & 1 & 3.33 & 5 & 13.89 & 13 & 13.13 & \\
\hline $\mathrm{Tx}$ & 0 & 0 & 0 & 0 & 1 & 2.78 & 1 & 1.01 & \\
\hline $\mathrm{N} 1$ & 5 & 15.15 & 7 & 23.33 & 6 & 16.67 & 18 & 18.18 & \\
\hline N2 & 15 & 45.45 & 12 & 40 & 20 & 55.56 & 47 & 47.47 & \\
\hline N3 & 7 & 21.21 & 10 & 33.33 & 5 & 13.89 & 22 & 22.22 & \\
\hline \multicolumn{10}{|c|}{ TNM, M status at diagnosis } \\
\hline Unknown & 0 & 0 & 1 & 3.33 & 0 & 0 & 1 & 1.01 & \\
\hline Mo & 33 & 100 & 29 & 96.67 & 36 & 100 & 98 & 98.99 & \\
\hline \multicolumn{10}{|l|}{ EGFR mutation status } \\
\hline Absent & 12 & 36.36 & 18 & 60 & 19 & 52.78 & 49 & 49.49 & \\
\hline \multicolumn{10}{|l|}{ ROS mutation status } \\
\hline Unknown & 22 & 66.67 & 15 & 50 & 21 & 58.33 & 58 & 58.59 & \\
\hline Absent & 11 & 33.33 & 15 & 50 & 15 & 41.67 & 41 & 41.41 & \\
\hline \multicolumn{10}{|l|}{ KRAS mutation status } \\
\hline Unknown & 23 & 69.7 & 12 & 40 & 18 & 50 & 53 & 53.54 & \\
\hline Absent & 9 & 27.27 & 15 & 50 & 10 & 27.78 & 34 & 34.34 & \\
\hline Present & 1 & 3.03 & 3 & 10 & 8 & 22.22 & 12 & 12.12 & \\
\hline \multicolumn{10}{|l|}{ BRAF mutation status } \\
\hline Unknown & 22 & 66.67 & 12 & 40 & 17 & 47.22 & 51 & 51.52 & \\
\hline Absent & 11 & 33.33 & 16 & 53.33 & 19 & 52.78 & 46 & 46.46 & \\
\hline Present & 0 & 0 & 2 & 6.67 & 0 & 0 & 2 & 2.02 & \\
\hline
\end{tabular}

Table 1 (continued) 
Table 1 (continued)

\begin{tabular}{|c|c|c|c|c|c|c|c|c|c|}
\hline \multirow{2}{*}{ Patient characteristics } & \multicolumn{6}{|c|}{ PD-L1 extent } & \multicolumn{2}{|c|}{ All } & \multirow{2}{*}{$P$ value } \\
\hline & $\mathrm{N}$ & $\%$ & $\mathrm{~N}$ & $\%$ & $\mathrm{~N}$ & $\%$ & $\mathrm{~N}$ & $\%$ & \\
\hline \multicolumn{10}{|l|}{ MET mutation status } \\
\hline Unknown & 23 & 69.7 & 14 & 46.67 & 17 & 47.22 & 54 & 54.55 & \\
\hline \multicolumn{10}{|l|}{ RET mutation status } \\
\hline Unknown & 24 & 72.73 & 18 & 60 & 24 & 66.67 & 66 & 66.67 & \\
\hline Absent & 8 & 24.24 & 12 & 40 & 12 & 33.33 & 32 & 32.32 & \\
\hline Present & 1 & 3.03 & 0 & 0 & 0 & 0 & 1 & 1.01 & \\
\hline Absent & 1 & 3.03 & 1 & 3.33 & 1 & 2.78 & 3 & 3.03 & \\
\hline \multicolumn{10}{|l|}{ ERBB2 mutation status } \\
\hline Unknown & 24 & 72.73 & 19 & 63.33 & 25 & 69.44 & 68 & 68.69 & \\
\hline Absent & 9 & 27.27 & 11 & 36.67 & 11 & 30.56 & 31 & 31.31 & \\
\hline \multicolumn{10}{|l|}{ Cancer progression } \\
\hline Yes & 18 & 54.55 & 18 & 60 & 6 & 16.67 & 42 & 42.42 & \\
\hline No & 15 & 45.45 & 12 & 40 & 30 & 83.33 & 57 & 57.58 & \\
\hline \multicolumn{10}{|c|}{$\begin{array}{l}\text { Treatment discontinued due to } \\
\text { progression }\end{array}$} \\
\hline Yes & 11 & 33.33 & 7 & 23.33 & 8 & 22.22 & 26 & 26.26 & 0.55 \\
\hline No & 22 & 66.67 & 23 & 76.67 & 28 & 77.78 & 73 & 73.74 & \\
\hline \multicolumn{10}{|c|}{$\begin{array}{l}\text { Treatment discontinued due to } \\
\text { other reasons }\end{array}$} \\
\hline Yes & 2 & 6.06 & 4 & 13.33 & 3 & 8.33 & 9 & 9.09 & 0.62 \\
\hline No & 31 & 93.94 & 26 & 86.67 & 33 & 91.67 & 90 & 90.91 & \\
\hline \multicolumn{10}{|l|}{ Living status } \\
\hline Living & 23 & 69.7 & 18 & 60 & 31 & 86.11 & 72 & 72.73 & \\
\hline Deceased & 10 & 30.3 & 12 & 40 & 5 & 13.89 & 27 & 27.27 & \\
\hline All & 33 & 100 & 30 & 100 & 36 & 100 & 99 & 100 & \\
\hline
\end{tabular}

$\mathrm{P}$ values by Fisher's exact test. Patients with unknown PD-L1 status were not included in this table. PD-L1, programmed-death ligand 1; TNM, tumor node metastases; EGFR, epidermal growth factor receptor oncogene; ALK, anaplastic lymphoma kinase oncogene; ROS, Ros oncogene; KRAS, KRAS oncogene; BRAF, BRAF oncogene; MET, MET oncogene; RET, RET oncogene; BRCA, breast cancer oncogene; ERBB2, ERBB2 oncogene. 


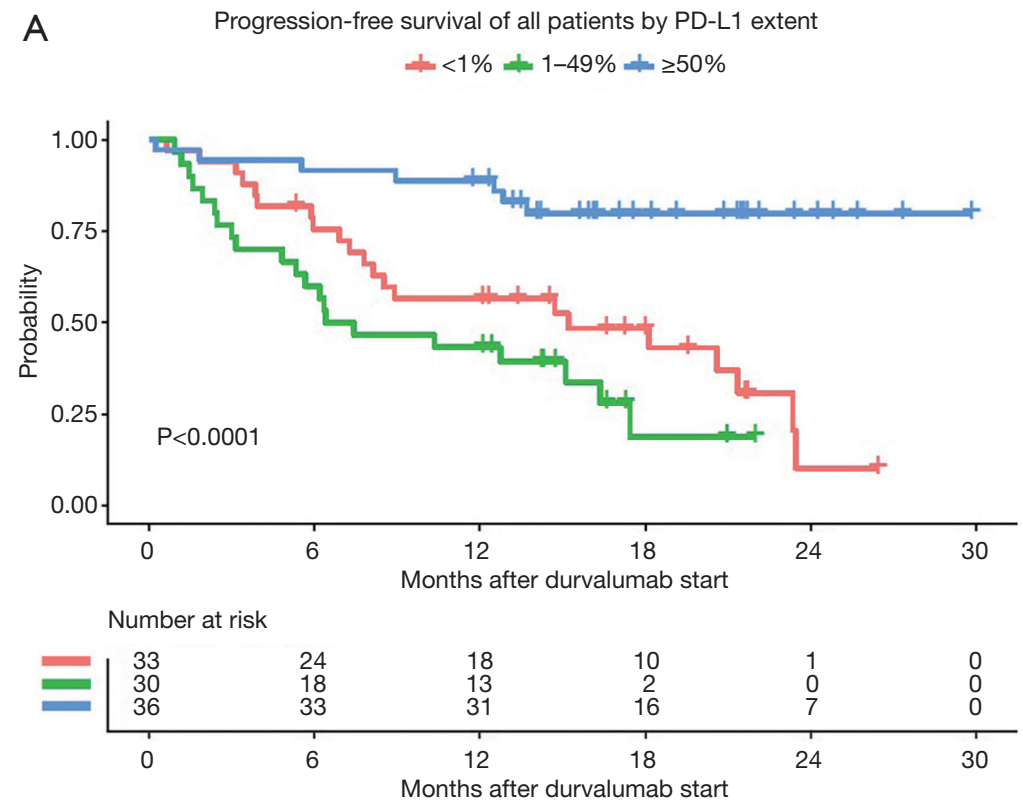

B Overall survival of all patients by PD-L1 extent

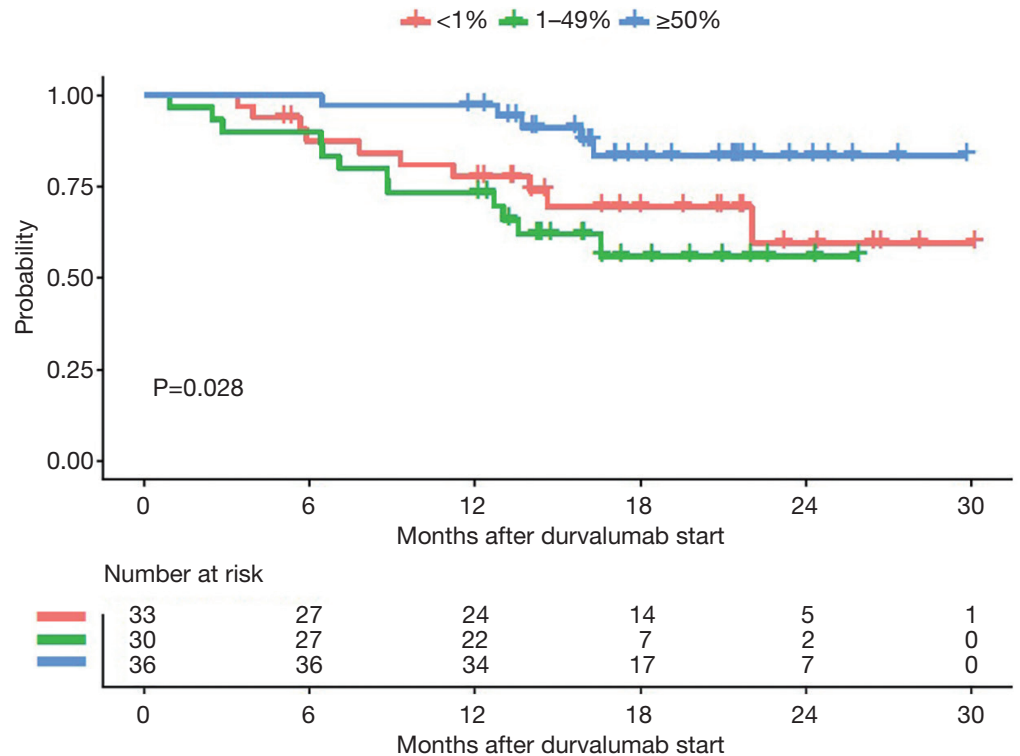

Figure 1 Kaplan-Meier curves of (A) progression-free survival and (B) overall survival by the extent of tumor PD-L1 expression. Survival was compared using log-rank testing between patient groups. PD-L1, programmed-death ligand 1.

in the $50-100 \%$ group (97\%) compared to the $1-49 \%$ group and the $<1 \%$ group $(73 \%$ and $78 \%$, respectively; $\mathrm{P}=0.028)$.

The median PFS for the group with $50-100 \%$ expression was 16.9 months, compared to 7.0 months for $1-49 \%$ expression group and 12.5 months for the $<1 \%$ expression group. The median OS was 17.6 months for the $50-100 \%$ expression group, 14.5 months for the 1-49\% expression group, and 14.8 months for the $<1 \%$ expression group. Survival analysis via Kaplan-Meier revealed a significant difference in both PFS (log-rank $\mathrm{P}<0.0001)$ and OS $(\log -$ rank $\mathrm{P}<0.028)$ based on the extent of PD-L1 expression (Figure 1). Multivariate analysis revealed that a PD-L1 expression $>50 \%$ was the only factor that was significantly 
Table 2 Summary of multivariate Cox proportional hazard model for PFS and OS

\begin{tabular}{lcccccc}
\hline & Factor & Comparison & Hazard ratio & $95 \%$ LCL & 95\% UCL & P value \\
\hline PFS & PD-L1 & $1-49 \%$ vs. $<1 \%$ & 1.446 & 0.752 & 2.777 & 0.2686 \\
& & $50-100 \%$ vs. $<1 \%$ & 0.205 & 0.086 & 0.491 & 17.096 \\
& & $50-100 \%$ vs. $1-49 \%$ & 7.037 & 2.896 & 0.0004 \\
OS & PD-L1 & $1-49 \%$ vs. $<1 \%$ & 1.289 & 0.535 & 0.176 & 0.973 \\
& & $50-100 \%$ vs. $<1 \%$ & 0.339 & 0.104 & 0.04 \\
& & $50-100 \%$ vs. $1-49 \%$ & 3.807 & 1.336 & 10.849 \\
\hline
\end{tabular}

Age and gender were forced into model to adjust their effects on PFS and OS. PD-L1 was significant after adjusting for them. PD-L1, programmed-death ligand 1; PFS, progression-free survival; OS, overall survival; LCL, lower control limit; UCL, upper control limit.

associated with improved PFS $(\mathrm{HR}=0.205, \mathrm{P}=0.0004)$ and OS ( $\mathrm{HR}=0.339, \mathrm{P}=0.04)$ when evaluated with age, sex, race, smoking status, the histologic subtype of NSCLC, tumor size and lymph node status (Table 2).

\section{Conclusions}

The results of our study demonstrate that the degree of tumor PD-L1 expression may predict the response of NSCLC patients to durvalumab therapy and that patients with $>50 \%$ PD-L1 expression had better outcomes and survived longer than those with $<50 \%$ expression. Notably, patients with PD-L1 expression of $1-49 \%$ and $<1 \%$ had relatively similar outcomes, which may explain why studies that used the cutoff of $<25 \%$ and $\geq 25 \%$ PD-L1 expression did not identify as significant a difference in outcomes (2). Limitations of our study include its retrospective design with potential resulting bias and our sample size of 121 patients, and external validity of our findings would require regular PD-L1 testing in the workup of NSCLC. Yet to our knowledge, this the largest observational study that showed a clear survival benefit favoring higher PD-L1 expression for stage III NSCLC patients undergoing durvalumab consolidation. These findings could potentially affect clinical decision-making regarding therapy selection and monitoring of these patients. More research is needed to determine the relationship between the extent of PD-L1 expression and the treatment outcomes in patients with locally advanced NSCLC. We recommend that future trials revolving around the use of immunotherapy, particularly durvalumab, in patients with NSCLC pay attention to the extent of PD-L1 expression and use the above cutoffs to better categorize patients and study their outcomes.

\section{Acknowledgments}

Funding: None.

\section{Footnote}

Reporting Checklist: The authors have completed the STROBE reporting checklist. Available at https://dx.doi. org/10.21037/tlcr-21-249

Data Sharing Statement: Available at https://dx.doi. org/10.21037/tlcr-21-249

Conflicts of Interest: All authors have completed the ICMJE uniform disclosure form (available at https://dx.doi. org/10.21037/tlcr-21-249). NAP has served as a consultant on advisory boards for Astrazeneca, Merck, BMS, Genentech, and Eli Lilly, with payments made to him. The other authors have no conflicts of interest to disclose.

Ethical Statement: The authors are accountable for all aspects of the work in ensuring that questions related to the accuracy or integrity of any part of the work are appropriately investigated and resolved. The study was conducted in accordance with the Declaration of Helsinki (as revised in 2013). The study was approved by the institutional review board of the Cleveland Clinic Foundation (NO: 14-562) and individual consent for this retrospective analysis was waived.

Open Access Statement: This is an Open Access article distributed in accordance with the Creative Commons Attribution-NonCommercial-NoDerivs 4.0 International License (CC BY-NC-ND 4.0), which permits the non- 
commercial replication and distribution of the article with the strict proviso that no changes or edits are made and the original work is properly cited (including links to both the formal publication through the relevant DOI and the license). See: https://creativecommons.org/licenses/by-nc-nd/4.0/.

\section{References}

1. American Cancer Society. Cancer Facts \& Figures 2021. Atlanta: American Cancer Society; 2021.

2. Antonia SJ, Villegas A, Daniel D, et al. Durvalumab after Chemoradiotherapy in Stage III Non-Small-Cell Lung Cancer. N Engl J Med 2017;377:1919-29.

3. Antonia SJ, Villegas A, Daniel D, et al. Overall Survival with Durvalumab after Chemoradiotherapy in Stage III NSCLC. N Engl J Med 2018;379:2342-50.

4. Paz-Ares L, Spira A, Raben D, et al. Outcomes with durvalumab by tumour PD-L1 expression in unresectable, stage III non-small-cell lung cancer in the PACIFIC trial. Ann Oncol 2020;31:798-806.

5. Stewart R, Morrow M, Hammond SA, et al. Identification and Characterization of MEDI4736, an Antagonistic Anti-PD-L1 Monoclonal Antibody. Cancer Immunol Res 2015;3:1052-62.

6. Postow MA, Callahan MK, Wolchok JD, et al. Immune Checkpoint Blockade in Cancer Therapy. J Clin Oncol 2015;33:1974-82.

7. Teixidó C, Vilariño N, Reyes R, et al. PD-L1 expression testing in non-small cell lung cancer. Ther Adv Med Oncol 2018;10:1758835918763493.

8. Garon EB, Rizvi NA, Hui R, et al. Pembrolizumab for the treatment of non-small-cell lung cancer. N Engl J Med 2015;372:2018-28.

9. Rizvi NA, Hellmann MD, Snyder A, et al. Cancer

Cite this article as: Jazieh K, Gad M, Saad A, Wei W, Pennell NA. Tumor PD-L1 expression is associated with outcomes in stage 3 NSCLC patients treated with consolidation durvalumab. Transl Lung Cancer Res 2021;10(7):3071-3078. doi: 10.21037/ tlcr-21-249 immunology. Mutational landscape determines sensitivity to PD-1 blockade in non-small cell lung cancer. Science 2015;348:124-8.

10. Spira AI, Park K, Mazières J, et al. Efficacy, safety and predictive biomarker results from a randomized phase II study comparing MPDL3280A vs docetaxel in $2 \mathrm{~L} / 3 \mathrm{~L}$ NSCLC (POPLAR). J Clin Oncol 2015;33:8010.

11. Herbst RS, Soria JC, Kowanetz M, et al. Predictive correlates of response to the anti-PD-L1 antibody MPDL3280A in cancer patients. Nature 2014;515:563-7.

12. Rizvi NA, Mazières J, Planchard D, et al. Activity and safety of nivolumab, an anti-PD-1 immune checkpoint inhibitor, for patients with advanced, refractory squamous non-small-cell lung cancer (CheckMate 063): a phase 2, single-arm trial. Lancet Oncol 2015;16:257-65.

13. Brody R, Zhang Y, Ballas M, et al. PD-L1 expression in advanced NSCLC: Insights into risk stratification and treatment selection from a systematic literature review. Lung Cancer 2017;112:200-15.

14. Rizvi NA, Brahmer JR, Ou S-HI, et al. Safety and clinical activity of MEDI4736, an anti-programmed cell deathligand 1 (PD-L1) antibody, in patients with non-small cell lung cancer (NSCLC). J Clin Oncol 2015;33:8032.

15. Antonia SJ, Balmanoukian A, Brahmer J, et al. Clinical Activity, Tolerability, and Long-Term Follow-Up of Durvalumab in Patients With Advanced NSCLC. J Thorac Oncol 2019;14:1794-806.

16. STROBE Statement: Observational Studies: Getting clear about transparency [Internet]. [cited 2021 Mar 9]. Available online: https://www.strobestatement.org/index.php?id=strobe-single-newsview\&tx_news_pi1\%5Bnews\%5D=146\&tx_news_ pi $1 \% 5 \mathrm{Bcontroller} \% 5 \mathrm{D}=$ News\&tx_news_pi1\%5Baction\% 5D=detail\&cHash=1313 eacdf5 efcb43416881ec91b8a1e6 